15 | 1993

Varia

\title{
Bertrand Barère, lecteur de Pascal
}

Présentation d'un commentaire inédit

Marie-Thérèse Bouyssy

\section{(2) OpenEdition}

Journals

Édition électronique

URL : http://journals.openedition.org/ccibp/600

DOI : $10.4000 /$ ccibp. 600

ISSN : 2493-7460

Éditeur

Centre international Blaise Pascal

\section{Édition imprimée}

Date de publication : 27 février 1993

Pagination : 11-13

ISSN : 0249-6674

Référence électronique

Marie-Thérèse Bouyssy, «Bertrand Barère, lecteur de Pascal », Courrier du Centre international Blaise Pascal [En ligne], 15 | 1993, mis en ligne le 07 janvier 2016, consulté le 04 mai 2019. URL : http:// journals.openedition.org/ccibp/600 ; DOI : 10.4000/ccibp.600

Ce document a été généré automatiquement le 4 mai 2019.

Centre international Blaise Pascal 


\title{
Bertrand Barère, lecteur de Pascal
}

\author{
Présentation d'un commentaire inédit
}

\author{
Marie-Thérèse Bouyssy
}

Le commentaire que nous présentons ici fut écrit en 1818 par Barère, l'ancien rapporteur du Comité de Salut public; celui qui fut la «voie de la Révolution", l'« Anacréon de la guillotine » selon une expression du temps, n'était plus alors qu'un « homme sans nom » à la manière du héros de Ballanche. Régicide rallié aux Cent-jours, il ne pouvait aucunement bénéficier de la loi dite d'amnistie du 12 janvier 1816. Réfugié à Mons et seul à vivre sous nom d'emprunt parmi les exilés qui trouvèrent asile aux Pays-Bas, il cherchait par une obscurité concertée à échapper à la vindicte des Bourbons et redoutait de possible excès de zèle des diverses polices françaises. À lui seul, il était devenu le souvenir vivant de la Révolution et de l'an II : il avait présidé le procès du roi, il avait scellé l'alliance de la Plaine et de la Montagne par son discours du 4 janvier 1793, ce qui avait conduit Louis XVI à l'échafaud, enfin, il n'avait cessé de rapporter sur tous les points les plus belles attentatoires à l'esprit de la Restauration, à savoir la mise en jugement de MarieAntoinette ou l'ordre de destruction des tombeaux des rois à Saint-Denis. De surcroît, il avait pu suivre à tous ces éléments "de tumulte et de fureur ", pour reprendre son propre langage.

Désormais condamné au loisir studieux, toujours vif d'esprit et de plume, Barère lit et consigne au fil des jours ses remarques sur l'actualité, la politique et l'histoire, le monde et ses injustices. Il dénonce avec constance les potentes improbi de l'heure. Il se consacre surtout à ses Senilia, du nom d'un néo-latinisme de sa création, des carnets de "mélanges et fragments, pensées, traits, extraits et souvenirs " comme l'annoncent les sous-titres de sa main. Il les qualifie également de "recherches, études, anecdotes, exquises et observations ». Pour la seule année 1818, les Archives Départementales des Hautes-Pyrénées possèdent ainsi au sein de leurs fonds Barère neuf carnets, soit 1120 feuillets qui représentent plus de six pages d'écriture quotidienne (et plus de trois selon notre norme à 1500 signes par page).

3 Barère inscrit la lecture parmi les arts libéraux, juste après la conversation qu'il aima et pratiqua avec virtuosité. Dans une énumération sur les «charmes de la vie civilisée », il cite dans l'ordre: «le travail agricole, l'industrie, la navigation, le commerce, la conversation, la 
lecture, la poésie, la musique, la danse, la liberté ${ }^{1}$ ». Il en fait fort classiquement une «source inépuisable pour l'esprit et la raison. C'est une grande ressource contre les malheurs de la vie ${ }^{2}$ » ou de façon plus intériorisée, il note qu'on ne peut se faire une bibliothèque de choix qu'après 40 ans : « on a quelque expérience de la vie et on a pu comparer en littérature le génie et le talent, l'inspiration et le goût, la philosophie et l'imagination. D'ailleurs, chacun choisit ses auteurs comme ses plaisirs et ses sociétés ${ }^{3}$ ». Déjà en l'an II, André Chénier, malgré le persiflage et le poids du contentieux politique, l'avait signalé comme «le plus savant » des sept cents bélittres de la Convention ${ }^{4}$. Son passé de publiciste l'avait de surcroît rendu lecteur averti et électrique. Dans les carnets chronologiquement proches de celui dont est extrait notre texte, on voit plus particulièrement affleurer les réflexions et citations libres de Voltaire et du prince de Ligne, du président Dupaty et de Chamfort. En 1817, son besoin de "penser l'histoire» sinon le présent l'a conduit à poursuivre de brouillonnes considérations sur les six premières Décades de Tite Live ${ }^{5}$. Cette séquence exceptionnellement longue, une trentaine de pages organisées en vingt points, livre pêlemêle les topoï sur la guerre et la paix et des remarques personnelles en matière de gouvernement. Il ne tente nullement d'habiller le présent à la romaine mais sa méditation vagabonde s'appuie sur un texte classique stricto sensu. La mémoire issue du collège nourrit l'anamnèse personnelle. La pensée en déroute puise de nouvelles ressources dans les textes archétypaux.

Le passage que nous présentons ici est extrait du carnet F 34.2 de juillet $1818^{6}$. L'histoire de la réception de Pascal est suffisamment lacunaire ${ }^{7}$ pour mériter attention :

L'éloquence élevée de Bossuet... frappe toujours et ne console jamais. Pascal est plus sublime quand il nous plaint en montrant à nu nos difformités. Il hait les vices mais on sent qu'il a des entrailles d'homme et qu'il s'afflige à notre sujet. Il a l'air de nous répéter sans cesse avec l'accent d'une piété véritable : «Infortunés, pourquoi vous faites-vous méchants, vous aviez tant besoin de vertu et de bonté pour ne pas aggraver le sort auquel notre vie est condamnée !». Quelquefois même, on devine que sa pensée n'est pas sortie tout entière de son cœur, qu'il ajoute en tremblant de commettre un blasphème: « 0 , mon Dieu! Pourquoi permettez-vous le vice? Comme le malheur de l'homme est-il entré dans les conseils de votre sagesse ». Il y a dans Pascal un génie qui perce tout, une lumière qui éclaire tout ce qu'il a voulu connaître et montrer. Il y a aussi des obscurités profondes et volontaires. Après avoir poussé l'examen de certaines choses plus loin qu'aucun autre penseur, on dirait qu'il s'est arrêté dans la crainte d'avoir des éblouissements de la raison et de la perdre même, en s'obstinant à étendre ses découvertes et de troubler toutes les certitudes qu'il croyait avoir acquises. Puis croyant et non moins investigateur que Bossuet, Pascal prouve, quand on le lit avec attention, qu'il a mesuré des yeux l'abîme du doute et qu'il s'est retiré des bords de cet abîme avec un effort dont l'impression n'a jamais su s'effacer entièrement. C'est alors que Pascal se réfugie dans sa foi comme dans un asile de paix où, délivré de ses terreurs, il puise de nouvelles forces pour annoncer des vérités qui lui paraissent utiles aux hommes. Le philosophe devient apôtre. Il juge l'homme et le monde comme s'il n'avait pas fait d'autre étude.

Ce texte ne peut se commenter sans rappeler la formule de Voltaire: "Ses Lettres provinciales... étaient un modèle d'éloquence et de plaisanterie. Les meilleures comédies de Molière n'ont pas plus de sel que les premières Lettres provinciales; Bossuet n'a rien de plus sublime que ces dernières ${ }^{8}$ ». Barère a une forme très différente d'être en dialogue avec le polémiste de Port-Royal. Il veut ignorer le jansénisme et le contexte, il surplombe cavalièrement la question du pari, il refuse de s'embarrasser des considérations usuelles en matière d'esprit de finesse et d'esprit de géométrie. Ces trois caractéristiques permettent à une 
volonté d'approche compréhensive de l'homme Pascal de s'exprimer plus librement. En lecteur aisé, Barère se dégage des conventions académiques pour en venir à ce qu'il revendique usuellement en matière de fraternité, le Nihil humanum a me alienum puto. Le Pascal de Barère est homme de passion. Il en saisit l'engagement dans la foi avec l'attention de celui-ci qui, dans un tout autre domaine, a défendu le pire et le plus terrible - sous la Terreur - tout en passant pour n'avoir d'autre conviction qu'opportuniste, une transcription possible du pari en termes laïcisés.

6 Dès lors, l'éblouissement de raison ruine l'esthétique de l'intelligence ${ }^{9}$ qui, raisons suffisantes et raisons militantes conjointes, conduisait à ne voir dans l'auteur des Pensées qu'un esprit exalté en proie à de pusillanimes superstitions. Le dialogue s'est construit ailleurs. Le sublime s'est investi de toute la fragilité humaine. Il n'est plus l'apanage du grand genre noble ${ }^{10}$. La génialité prométhéenne de l'homme s'est d'elle-même investie de l'effroi qui marque toute conscience réflexive ${ }^{11}$. Ce n'est pas là pusillanimité mais le chemin par où l'on peut considérer qu'il pascalise en certains de ses textes assez personnels de la fin des années 1820 :

Le voyageur surpris.

Une lampe éclaire un voyageur dans sa course de nuit, au milieu d'un pays inconnu. Sa lampe vient à s'éteindre. Le voyageur éperdu, épouvanté du désert où il est forcé de s'arrêter dans une nuit profonde réfléchit un instant; il regarde autour de lui, plein de doutes et d'effroy. Bientôt, il adresse sa prière au dieu de la nature. Les ténèbres se dissipent. Une marche à la lueur des étoiles. Le firmament plein des merveilles du créateur a aussi sa lumière. Le voyageur tombe à genoux, le cœur rempli de reconnaissance pour la divinité, heureux de découvrir qu'aucune lampe ne vaut la lumière qui descend du ciel ${ }^{12}$.

7 Ici se combinent éléments kantiens et atmosphère à la Füssli sur fond de désenchantement $d u$ monde. Le dieu de la nature est celui $d u$ XVII ${ }^{e}$ siècle mais l'effondrement de l'optimisme suscite la résurgence quasi fidéiste des plus vieux thèmes augustiniens. Est-ce à dire que l'inquiétude romantique est celle d'un Pascal et que l'on put en faire alors un "René de la téléologie »? Ce serait allé trop vite en besogne. Certes, Barère fut croyant à la manière de son siècle. Hippolyte $\operatorname{Carnot}^{13}$, son premier biographe, le dit. Des écrits échelonnés tout au long de sa très longue vie, avant et après la Révolution, en témoignent. Il récusa l'athéisme avec constance :

De l'athée.

De moi, an 6. Le système de l'athée et ses prétextes de ne pas croire sont comme des jalons plantés de loin en loin sur une route obscure et escarpée de ces jalons ne mènent qu'à un désert aride, le néant. La morale de l'immortalité et sa croyance ressemble à un pays entrecoupé de montagnes et de rochers mais qui, à travers le désert de la vie, amène dans une vallée fertile, délicieuse où tous les êtres bons, sensibles, généreux, bienfaisants et utiles sont rassemblés. Il est facile de choisir entre les deux opinions (F 46).

mais il écourte le débat non sans désinvolture :

Quand on peut se perdre dans des ténébreuses profondeurs de la métaphysique, il faut se condamner à lire Aristote, Malebranche, Leibnitz, Barclay [sic], Priestley et autres auteurs de ce genre si peu satisfaisants pour la raison et si stériles pour la société (F 34.1).

Car la seconde génération des Lumières a définitivement substitué le sentiment aux questionnements antérieurs. Le débat ne peut même plus se soutenir d'une attention narquoise à la manière de Voltaire. Les apologies sont désormais les auteurs sensibles $d u$ siècle. Ils ne sont ni beaux esprits ni théologiens : 
Pour avoir une juste idée d'un philosophe chrétien, Fénelon au théâtre, le curé de Mélanie, drame de la Harpe, philosophe catholique apostolique et romain. Lisez aussi la belle confession du vicaire savoyard dans l'Émile de Rousseau ${ }^{14}$. par Racine (le fils ${ }^{15}$ ). Cela ne périme en rien les horizons plus anciens de la sensibilité commune tant il est vrai qu'une culture se joue dans le dialogue de longue durée qui traverse ses horizons feuilletés.

L'attention que Barère porte à Pascal est donc celle d'un moment. Elle est tout entière consacrée à l'homme inquiet dans l'ordre de la charité (soit de la foi). Elle pénètre avec esprit de finesse peut-on dire, humaniste chrétien et vigilant. André Suarez ironisait sur la capacité qu'à chaque temps de "travestir Pascal à sa mode ${ }^{16}$ ». Le mérite de ce texte est d'en témoigner avec la facilité propre à Barère, celle de l'improvisateur politique qui sait dans l'instant «embrasser l'ensemble de l'état social et se saisir de l'imagination intérieure des sociétés ${ }^{17}$ "Sans abdiquer en rien des options du siècle des Lumières, à l'écoute des nouvelles donnes d'une époque qui n'a plus confiance dans le triomphalisme de la seule raison, une façon d'en revenir comme en sourdine aux problèmes du pari, il se saisit d'abord des certitudes de la très vieille culture humaniste chrétienne. Les esprits sceptiques peuvent n'y voir qu'un réservoir de clichés mais par-delà la rhétorique des mises en posture, les textes, à condition de bien vouloir les écouter, laissent entrevoir combien ces éléments restent actifs et sensibles.

\section{NOTES}

1. Carnet $\mathrm{F} 34.4$ des Senilia, octobre 1818. Toutes nos références à des textes manuscrits et inédits renvoyant au fonds Barère des Archives Département des Hautes-Pyrénées, nous ne donnons que la cote au sein de ce fonds.

2. Carnet F 35.15, fin 1820.

3. F 35.3, janvier 1818.

4. Cuvres complètes, Paris, 1989, Iambes VIII, p. 193.

5. Cf. notre article «Barère, la lecture et la Bibliothèque nationale » in Revue de la Bibliothèque nationale, hiver 1990, pp. 52-60.

6. In ma thèse "Trente ans après, Bertrand Barère sous la Restauration ou la rhétorique du Ténare ", Paris I, 1993, p. 247-249.

7. Cf. Jean Ehrard. "Pascal au siècle des Lumières " in Pascal présent, 1662-1962, ClermontFerrand, 1963, pp. 231-255.

Jean Mesnard. « Voltaire et Pascal » in La culture du XVII siècle, enquêtes et synthèses, Paris, 1992, pp. 589-599 (article publié à l'origine en 1979).

8. Le siècle de Louis XIV, chap. XXXVII, Du jansénisme.

9. Notion reprise de Jacques Chouillet, L'esthétique des Lumières, Paris, 1974, p. 4-6.

10. À preuve l'usage que Barère fait du mot dans son Salon imaginaire ou le $20^{e}$ siècle, 230 notices rédigées dans l'esprit de celles des salons de peinture du temps. Il réserve la notion au seul portrait du Christ par Michel-Ange (215, après 1830). 
11. Dans le même Salon imaginaire, notice 74, Phidias, l'image de la génialité modeste, reste « épouvanté de son propre ouvrage ».

12. Album d'esquisses, $\mathrm{F} 76, \mathrm{f}^{\circ} 119$. On ne peut que mettre en regard de ce texte la pensée 693 (éd. Brunschvicg) devenue $n^{\circ} 229$ in éd. Philippe Sellier.

13. Son exécuteur testamentaire qui publia ses Mémoires post-mortem conformément à un arrangement prévu en 1838. Hippolyte Carnot, saint-simonien et fils du grand Carnot, faisait figure d'héritier légitime du jacobinisme et avait rencontré le vieux Barère.

14. Senilia, F 35.11.

15. Senilia, F 39.3.

16. Puissance de Pascal, Paris, 1923, p. 89.

17. Senilia F. 35.15, 1820.

INDEX

Mots-clés : Pascal, Barère (Bertrand), commentaire

Keywords : Pascal, Barère (Bertrand), comment

\section{AUTEUR}

\section{MARIE-THÉRÈSE BOUYSSY}

U.R.A. 96 Langues et littératures françaises,

$17 \mathrm{e}$ et $18 \mathrm{e}$ siècles (CNRS., Paris IV) 